\title{
Detection of Horseradish (Armoracia rusticana) Myrosinase Genes in Samples
}

\section{Containing Horseradish}

\author{
Masakazu Hara ${ }^{1 *}$, Masaki Oda ${ }^{1}$, Tokiyasu Yogo ${ }^{1}$, Tatsuyoshi SumI ${ }^{2}$, Ryo AraI ${ }^{2}$, Toru Kubor ${ }^{1}$ and Hideo Etoh ${ }^{1}$ \\ ${ }^{1}$ Faculty of Agriculture, Shizuoka University, 836 Ohya, Suruga-ku, Shizuoka 422-8529, Japan \\ ${ }^{2}$ Research and Development Division, S\&B FOODS INC., 38-8 Miyamoto-cho, Itabashi-ku, Tokyo 174-8651, Japan
}

Received December 20, 2007; Accepted April 10, 2008

Horseradish (Armoracia rusticana) is a traditional herb used mainly in Asia, Europe, and North America. Processed food products that include horseradish as an ingredient have been developed and produced. Here, we report on the detection of multiple myrosinase genes of horseradish that closely resemble each other. For amplification of each myrosinase gene, a primer was designed and subjected to polymerase chain reaction (PCR) to obtain the horseradish DNA fragments (ca. 500 bp). PCR using the myrosinase gene primers obtained no corresponding DNA fragments of other species, including wasabi, radish, spinach, and pea. This amplification technique is therefore applicable for the specific detection of horseradish for processed food products.

Keywords: Armoracia rusticana, horseradish, myrosinase

\section{Introduction}

Horseradish (Armoracia rusticana) is a perennial herb of the Brassicaceae family that is grown in Asia, Europe, and North America. Processed food products, such as horseradish dry powder, horseradish paste and prepared horseradish mixed with other condiments, have been developed and produced. Recently, reported health benefits of horseradish and its constituents include plasma cholesterol reduction in mice (Balasinska et al., 2005), tumor cell proliferation and cyclooxygenase activity prevention (Weil et al., 2005), as well as an antimicrobial effect on Vibrio parahaemolyticus by horseradish extract (Yano et al., 2006). Allyl isothiocyanate, a major pungent component in horseradish ( $\mathrm{Li}$ and Kushad, 2005; Zhang et al., 2007), shows biological activities, such as inhibition of urotoxicity in mice (Manesh and Kuttan, 2005), bactericidal activities (Nadarajah et al., 2005; Shin et al., 2004), and antifungal activity (Mari et al., 2002).

Isothiocyanates are generated by a two component myrosinase-glucosinolate system (Rask et al., 2000). The mechanical disruption of plant tissue provides the enzymatic reaction, which converts glucosinolates to the corresponding isothiocyanates. Myrosinase genes have been isolated

*To whom correspondence should be addressed.

Email: masahara@agr.shizuoka.ac.jp from Brassicaceae plants (Thangstad et al., 1993). From the amino acid sequences, myrosinases of Brassicaceae fall into two major subfamily types, the Brassicaceae crop type and Arabidopsis type (Saitoh et al., 2006). However, myrosinases of horseradish (Li et al., 2007) and wasabi (Eutrema wasabi) (Saitoh et al., 2006) do not fall into either subfamily type.

Food companies have developed processed food products related to horseradish. Since information about the composition of processed food products has become important, the inclusion of a universal method to detect horseradish is necessary. In the case of wasabi, which is a pungent condiment used in Japanese dishes, a polymerase chain reaction (PCR)based detection method with specific primers to amplify wasabi myrosinase genes from products containing wasabi has been reported by Hara et al. (2007). In this paper, we apply a similar method to detect horseradish myrosinase genes in products containing horseradish.

\section{Materials and Methods}

Plant materials Two cultivars of horseradish (Armoracia rusticana), a Bohemian type and a common type, were grown in a field at S\&B Foods Inc., Japan and were harvested after 1 year. The petioles were kept at $-70^{\circ} \mathrm{C}$ until analysis. Radish, spinach and peas were purchased at a local 
market in Japan.

Food products Horseradish powder and ready-to-use wasabi paste were purchased from a food market in Japan and kept at $4{ }^{\circ} \mathrm{C}$ until DNA extraction analysis.

Cloning of myrosinase cDNA fragments Total RNA was extracted from the horseradish petiole with an RNeasy Plant Mini Kit (Qiagen, Tokyo, Japan). Petiole tissues were used for the RNA extraction. The petiole was preferred over the root because a better quality of RNA could be extracted. Early experiments unintentionally found partial sequences of two cDNA clones, which were selected from reverse transcription-polymerase chain reaction (RT-PCR) products amplified from the horseradish petiole, and were identical to the previously reported root-derived horseradish myrosinase sequence, ArMY1 (accession No. AY822710, Li et al., 2007). This finding showed that expression of myrosinase could be similar for both the petiole and the root of the horseradish. The cDNA fragments of horseradish myrosinase were prepared by RT-PCR using an RNA PCR Kit ver. 2.1 (Takara, Tokyo, Japan). The two cultivars were labeled "Bohemian" and “common”. Total RNA (1 mL) was reverse transcribed at $42^{\circ} \mathrm{C}$ for $30 \mathrm{~min}$, and PCR with the primers, Myr1 and Myr3 (Fig. 1), was performed 40 cycles of $94^{\circ} \mathrm{C}$ for $30 \mathrm{~s}$, $45^{\circ} \mathrm{C}$ for $30 \mathrm{~s}$, and $72^{\circ} \mathrm{C}$ for $120 \mathrm{~s}$. The PCR products (ca. $700 \mathrm{bp}$ ) were cloned into a pDrive Cloning vector (PCR Cloning Kit, Qiagen). Nucleotide sequencing was performed using a SequiTherm EXCEL II Long-Read Premix DNA
Sequencing Kit-LC (Epicenter Technologies, Tokyo, Japan) on a DNA sequencer (Model 4000L, Aloka, Tokyo, Japan). Alignment and phylogenetic analysis implemented the unweighted pair group method using arithmetic average (UPGMA) and GENETYX-MAC Ver. 8.0 software (GENETYX, Tokyo, Japan).

Preparation of pastes Rhizome of horseradish and wasabi paste were both ground by a steel musher until forming a smooth paste. The pastes were mixed with the following paste ratios (w/w): 0\%, 0.01\%, 0.1\%, 1\%, 5\%, 10\%, and $100 \%$ of horseradish. Corresponding pastes (100 mg each) were frozen by liquid $\mathrm{N}_{2}$ and kept at $-70^{\circ} \mathrm{C}$ until analysis.

Detection of horseradish myrosinase genes DNA of horseradish, wasabi, radish, spinach, and pea plants were extracted by a DNeasy Plant Mini Kit (Qiagen). Pastes and processed food products were directly extracted. After extraction, quality of the DNA fractions was estimated by using $0.5 \%$ agarose gel electrophoresis. DNA samples $(0.5 \mu \mathrm{g})$ were used for PCR templates. Taq DNA polymerase used was Gene Taq (Nippon Gene, Tokyo, Japan). PCR with the primers, Seiwasa1 and Seiwasa3 (Fig. 1), was performed for either 30 (Fig. 3A) or 25 (Fig. 4) cycles of $94^{\circ} \mathrm{C}$ for $30 \mathrm{~s}, 60^{\circ} \mathrm{C}$ for $30 \mathrm{~s}$, and $72^{\circ} \mathrm{C}$ for $120 \mathrm{~s}$. PCR products were analyzed by $1 \%$ agarose gel electrophoresis. For nested PCR, 10 ng of the initial 30-cycle PCR products were reamplified under the same cycle conditions with primers, Seiwasa2 and Seiwasa3 (Fig. 1).

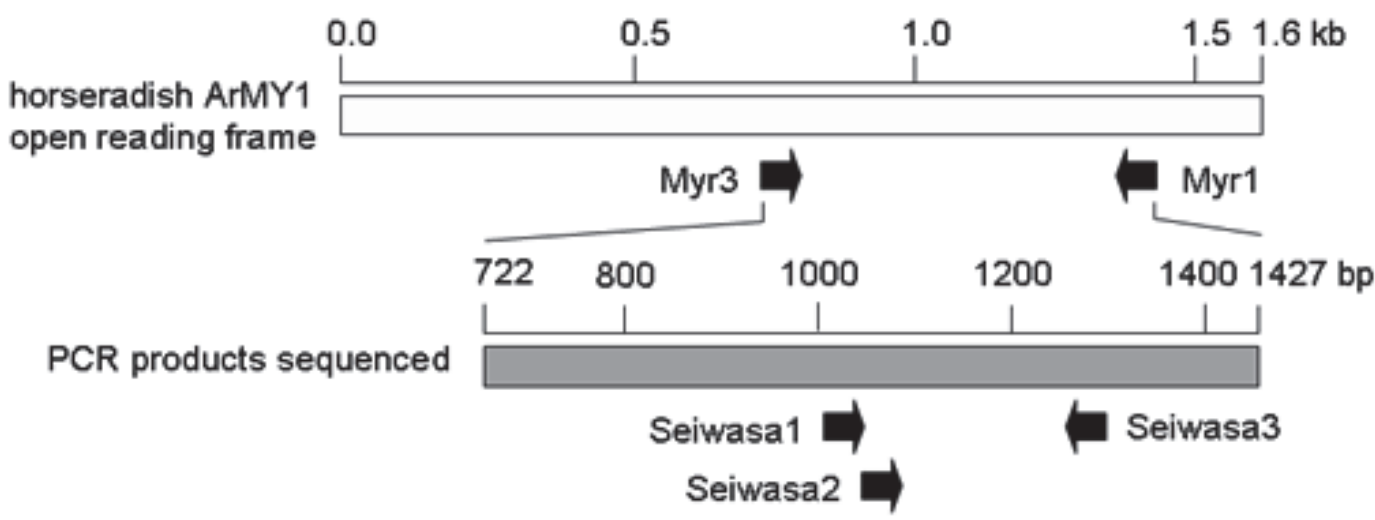

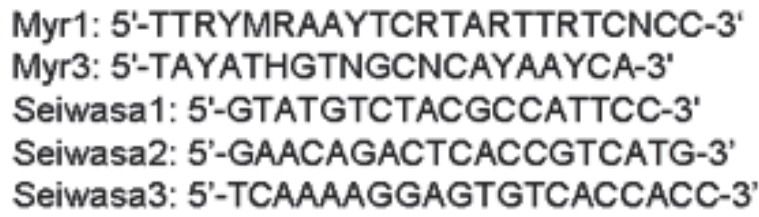

Myr1: 5'-TTRYMRAAYTCRTARTTRTCNCC-3' Myr3: 5'-TAYATHGTNGCNCAYAAYCA-3 Seiwasa2: 5'-GAACAGACTCACCGTCATG-3' Seiwasa3: 5'-TCAAAAGGAGTGTCACCACC-3'

Fig. 1. Positions and sequences of primers for myrosinase genes.

Right- and left-directed arrows indicate sense and antisense primers, respectively. Locations of the arrows in the figure are approximate. Numbers on the right side of each nucleic acid sequence show a position of the corresponding primer. The numbers represent base pairs from the 5' end of the ArMY1 open reading frame. 


\section{Results and Discussion}

First, the differences between horseradish myrosinase and other myrosinases were investigated to determine whether the gene of horseradish myrosinase was useful for specific detection of horseradish. Plant myrosinases are generally classified into the Brassicaceae crop type and the Arabidopsis type. Recently, sequences of wasabi myrosinase WjMY1 (accession number AB194903) (Saitoh et al., 2006) and horseradish myrosinase ArMY1 (accession number AY822710) (Li et al., 2007) have been determined. Hara et al. (2007) reports that the homology between ArMY1 and other myrosinases range from $75.7 \%$ (ArMY1 vs. radish MB) to $77.5 \%$ (ArMY1 vs. Arabidopsis TGG2). Because the nucleic acid sequences between ArMY1 and other myrosinases are less than $22.5 \%$ different, we predict that distinction between horseradish myrosinase(s) and other plant myrosinases is possible by PCR.

Little information is known about the constitution of a gene family of myrosinase in horseradish. Thus, the variation of myrosinase genes in horseradish was investigated by RT-PCR with degenerate primers, Myr1 and Myr3 (Fig. 1), which were designed from conserved regions of the myrosinase amino acid sequences among the Brassicaceae crop type and the Arabidopsis type (Hara et al., 2000). As was expected, the exclusive amplification of myrosinase genes was expressed from the horseradish. From the Bohemian and common cultivar types tested, the expected cDNA fragments (ca. $700 \mathrm{bp}$ ) were amplified from the RNA samples (data not shown). After cloning the fragments into the plasmid vector, we sequenced 13 clones (7 clones in Bohemian and 6 clones in common). The clone names were Bohemian1, Bohemian2, Bohemian3, Bohemian4, Bohemian5, Bohemian7 and Bohemian9, and common1, common2, common3, common5, common6, and common8. All 13 clones had a nucleic acid length of $706 \mathrm{bp}$. Bohemian1 (663 bp) with both ends of the PCR products corresponding to the degenerate primers eliminated was shown in Fig. 2. A sequence analysis demonstrated that 6 out of the 13 clones, (Bohemian1, Bohemian2, Bohemian9, common1, common6, and common8) had identical sequences. The sequences of the 6 clones were also identical to the corresponding sequence in the horseradish myrosinase clone, ArMY1 (accession number AY822710),

$\mathrm{a} \quad \mathrm{b}$

$\mathrm{C}$

001: СTTCTTGCTCATGCCACGGCCGTTGATCTTTACAGGABGAAMTATAAGÄGGÄAGGGGGGACAAATCGGACCTGTGATGATAACAAGATGGTTTCTTC: 100 d

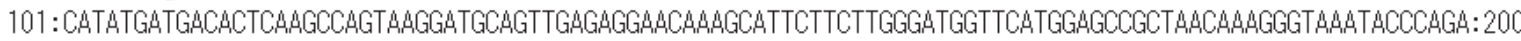

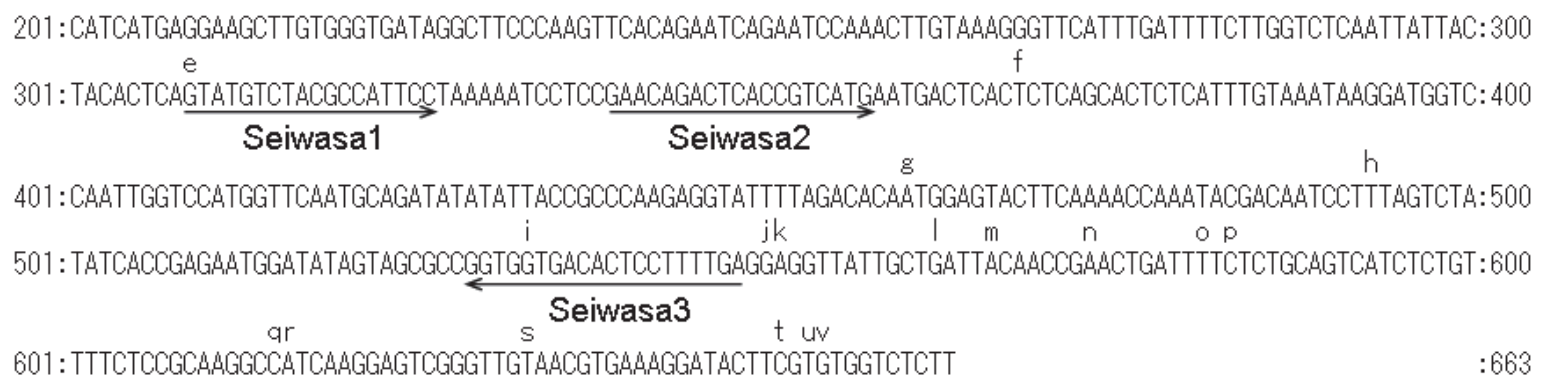

\begin{tabular}{|c|c|}
\hline clone & mutations \\
\hline Bohem ian3 & 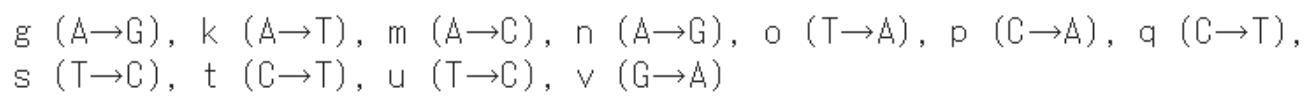 \\
\hline Bohemian 4 & $r(A \rightarrow G)$ \\
\hline Bohemian5 & $a(G \rightarrow A)$ \\
\hline Bohemian 7 & $d(T \rightarrow C), \quad f(T \rightarrow C), \quad \mid \quad(G \rightarrow A)$ \\
\hline common'2 & $b(T \rightarrow C)$ \\
\hline $\begin{array}{l}\text { common3 } \\
\text { common5 }\end{array}$ & $e(B \rightarrow C), h(T \rightarrow C), \quad i \quad(G \rightarrow A)$ \\
\hline common5 & $c(A \rightarrow G), \quad j \quad(G \rightarrow A)$ \\
\hline
\end{tabular}

Fig. 2. Nucleic acid sequence of a Bohemian1 clone and mutations found in other clones.

Nucleic acid sequence of a Bohemian1 clone is shown above. Small letters (a-v) represent positions of mutations. PCR primers (Seiwasa1, Seiwasa2, and Seiwasa3) are represented by arrows at the corresponding sites. Seiwasa1 and Seiwasa2 are sense primers. Seiwasa3 is an antisense primer. Mutations in 7 clones are summarized below (i.e., "g $(A \rightarrow G)$ )" means that $\mathrm{A}$ in the Bohemian1 clone changes to $\mathrm{G}$ at the position of $\mathrm{g}$. 
which was obtained from a horseradish root (Li et al., 2007). This result showed that ArMY1 could be a myrosinase clone mainly expressing in the horseradish plant. The other 7 clones were not identical, but similar to Bohemian1. The greatest variation to the Bohemian1 was Bohemian3 with 98.3\% homology. This result suggests that horseradish may possess a large myrosinase gene family consisting of multiple copies of genes with minor mutations.

To perform sensitive detection of horseradish myrosinase genes, 2 primers, Seiwasa1 and Seiwasa3, were prepared that were could amplify all 13 clones (Figs. 1, 2). The sequences of Seiwasa1 and Seiwasa3 could not be found in the sequence of wasabi myrosinase WjMY1 (data not shown), thus it was likely that the two primers could not anneal to the wasabi myrosinases. DNA samples were extracted from the Bohemian type and the common type. When the DNA samples were subjected to PCR with primers, Seiwasa1 and Seiwasa3, an apparent band at a calculated 500 bp was found in each reaction (Fig. 3A). However, no visible signals appeared in the DNA samples of wasabi, radish, spinach, or pea. The fragment size amplified with Seiwasa1 and Sei- wasa3 was calculated to be $239 \mathrm{bp}$, excluding introns. However, the bands indicated in Fig. 3A are significantly larger than the expected size. To test whether the bands amplified from horseradish DNA were fragments of myrosinase genes, nested PCR was performed. PCR products amplified by using Seiwasa1 and Seiwasa3 were successfully re-amplified by Seiwasa2 and Seiwasa3 (Fig. 3B, lane 1 for the Bohemian type and lane 3 for the common type). The re-amplification demonstrated that the 500-bp fragments, which could contain intron(s), were portions of horseradish myrosinase genes. Two additional bands were co-amplified by the PCR (Fig. 3). The sizes were calculated to be $740 \mathrm{bp}$ and $240 \mathrm{bp}$. These minor bands were usually fainter than the major band (ca. 500 bp). The larger band (ca. 740 bp) seemed to be specific to horseradish, but the smaller one (ca. 240 bp) appeared nonspecifically in all samples tested. It was assumed that the major band is not disturbed by the additional bands as the sizes of the bands were totally different.

Whether the detecting method could be applied to horseradish-related processed food products was also tested. In Japan, horseradish-related food products frequently contain
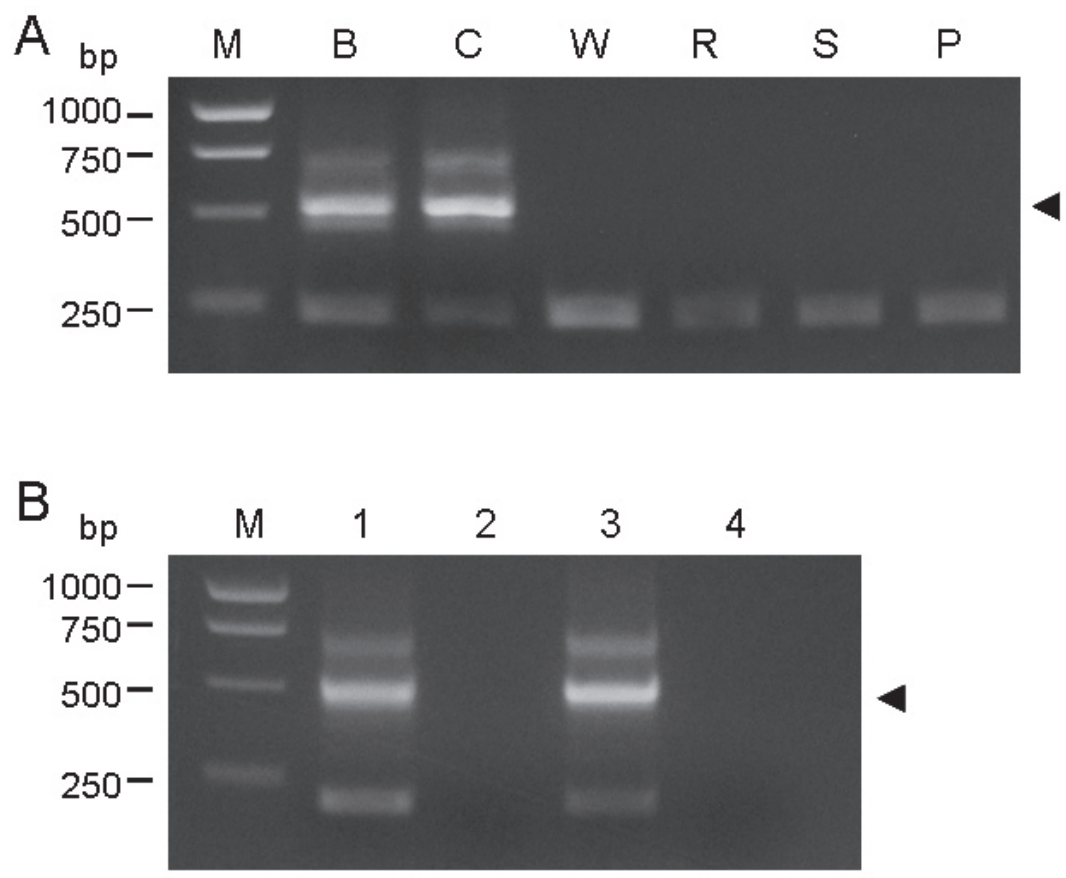

Fig. 3. Amplification of myrosinase gene fragments from horseradish (A. rusticana) DNA. (A) Results of PCR with primers Seiwasa1 and Seiwasa3. DNA samples are prepared from horseradish cultivars and other plants. The PCR conditions are described in the Materials and Methods section. Lane M, DNA size marker; lane B, horseradish Bohemian type; lane C, horseradish common type; lane W, wasabi (Eutrema wasabi); lane R, radish (Raphanus sativus); lane S, spinach (Spinacia oleracea); lane P, pea (Pisum sativum). An arrowhead indicates apparent bands found in horseradish cultivars. (B) Nested PCR by a combination of Seiwasa2 and Seiwasa3. Lane M, DNA size marker; lane 1, horseradish Bohemian type with re-amplification; lane 2, horseradish Bohemian type without re-amplification; lane 3, horseradish common type with re-amplification; lane 4, horseradish common type without reamplification. An arrowhead indicates expected bands. 


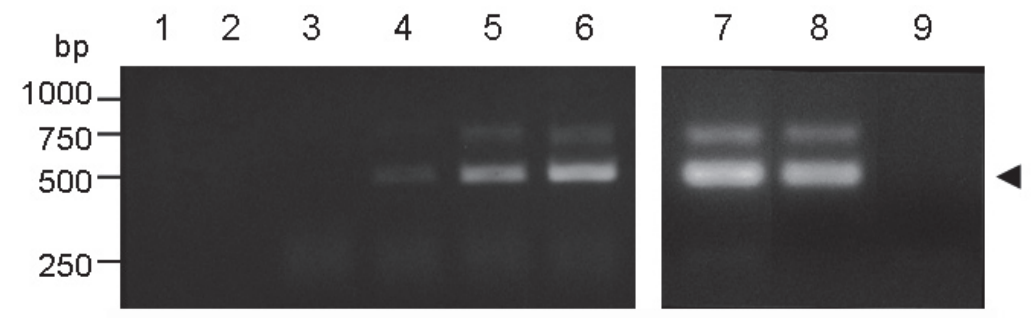

Fig. 4. Detection of myrosinase genes in pastes and food products. The PCR conditions are described in the Materials and Methods section. Primers Seiwasa1 and Seiwasa3 are used. Lane 1, pure wasabi paste; lane 2, mixed paste with $0.01 \%$ of horseradish paste $(\mathrm{w} / \mathrm{w})$; lane 3 , mixed paste with $0.1 \%$ of horseradish paste $(\mathrm{w} / \mathrm{w})$; lane 4 , mixed paste with $1 \%$ of horseradish paste $(\mathrm{w} / \mathrm{w})$; lane 5 , mixed paste with $5 \%$ of horseradish paste (w/w); lane 6, mixed paste with $10 \%$ of horseradish paste (w/w); lane 7, 100\% of horseradish paste; lane 8 , horseradish powder; lane 9 , ready-to-use wasabi paste.

not only horseradish but also wasabi; thus, mixed pastes consisting of horseradish and wasabi in various proportions were prepared, as shown in the legend of Fig. 4. The PCR condition was the same as that the conditions in Fig. 3A. Apparent bands (ca. 500 bp) were obtained in the pastes containing horseradish at $5 \%(\mathrm{w} / \mathrm{w}), 10 \%(\mathrm{w} / \mathrm{w})$, and $100 \%(\mathrm{w} / \mathrm{w})$ (lanes 5, 6 and 7 in Fig. 4). A faint band was detected in the paste containing $1 \%(\mathrm{w} / \mathrm{w})$ of horseradish (lane 4 in Fig. 4). However, no visible signals were found in the pastes containing $0 \%(\mathrm{w} / \mathrm{w}), 0.01 \%(\mathrm{w} / \mathrm{w})$, and $0.1 \%(\mathrm{w} / \mathrm{w})$ of horseradish (lanes 1, 2 and 3 in Fig. 4). These results suggest that a detection limit for horseradish in the paste was a calculated $1 \%$ in the present condition. Two kinds of commercial food products, horseradish powder and a ready-to-use wasabi paste, purchased from a food market in Japan were then tested. The powder contained horseradish, while the wasabi paste did not. A clear band appeared in the horseradish powder (lane 8, Fig. 4), but no signal was indicated in the readyto-use wasabi paste (lane 9, Fig. 4). These results suggest that the present method can detect genes for horseradish myrosinase from products containing horseradish.

Processed food products containing horseradish have been produced as ready-to-use seasonings. Although consumer concerns about the composition of food products are increasing, techniques for detecting horseradish have not significantly improved. Here, we propose a method for detecting horseradish myrosinase genes from samples containing horseradish. This method is based on PCR techniques with the use of specific primers for horseradish myrosinases. The specific primers, Seiwasa1 and Seiwasa3, can anneal to corresponding sites of myrosinase genes forming multiple copies in horseradish DNA. Thus, selective and sensitive detection of myrosinase is possible. As detection of the horseradish myrosinase genes in the horseradish paste and the horseradish powder has been performed here, testing for horseradish in food products is now possible.

\section{References}

Balasinska, B., Nicolle, C., Gueux, E., Majewska, A., Demigne, C. and Mazur, A. (2005). Dietary horseradish reduces plasma cholesterol in mice, Nutrition Res., 25, 937-945.

Hara, M., Fujii, Y., Sasada, Y. and Kuboi, T. (2000). cDNA cloning of radish (Raphanus sativus) myrosinase and tissue-specific expression in root, Plant Cell Physiol., 41, 1102-1109.

Hara, M., Yogo, T., Sumi, T., Arai, R., Kuboi, T. and Etoh, H. (2007). Detection of wasabi (Wasabia japonica Matsum.) in food products by using myrosinase genes, Food Sci. Technol. Res., 13, 380-384.

Li, X. and Kushad, M.M. (2005). Purification and characterization of myrosinase from horseradish (Armoracia rusticana) roots, Plant Physiol. Biochem., 43, 503-511.

Li, X., Wen, Z., Bohnert, H.J., Schuler, M.A. and Kushad, M.M. (2007). Myrosinase in horseradish (Armoracia rusticana) root: Isolation of a full-length cDNA and its heterologous expression in Spodoptera frugiperda insect cells, Plant Sci., 172, 1095-1102.

Manesh, C. and Kuttan, G. (2005). Effect of naturally occurring isothiocyanates in the inhibition of cyclophosphamide-induced urotoxicity, Phytomedicine, 12, 487-493.

Mari, M., Leoni, O., Iori, R. and Cembali, T. (2002). Antifungal vapour-phase activity of allyl-isothiocyanate against Penicillium expansum on pears, Plant Pathol., 51, 231-236.

Nadarajah, D., Han, J.H. and Holley, R.A. (2005). Inactivation of Escherichia coli O157:H7 in packaged ground beef by allyl isothiocyanate, Int. J. Food Microbiol., 99, 269-279.

Rask, L., Andreasson, E., Ekbom, B., Eriksson, S., Pontoppidan, B. and Meijer, J. (2000). Myrosinase: gene family evolution and herbivore defense in Brassicaceae, Plant Mol. Biol., 42, 93-113.

Saitoh, S., Hara, M., Kuboi, T. and Etoh, H. (2006). Cloning of 
myrosinase cDNA from Wasabia japonica Matsum, Food Sci. Technol. Res., 11, 412-415.

Shin, I.S., Masuda, H. and Naohide, K. (2004). Bactericidal activity of wasabi (Wasabia japonica) against Helicobacter pylori, Int. J. Food Microbiol., 94, 255-261.

Thangstad, O.P., Winge, P., Husebye, H. and Bones, A. (1993). The myrosinase (thioglucoside glucohydrolase) gene family in Brassicaceae, Plant Mol Biol., 23, 511-524.

Weil, M.J., Zhang, Y. and Nair, M.G. (2005). Tumor cell proliferation and cyclooxygenase inhibitory constituents in horseradish
(Armoracia rusticana) and wasabi (Wasabia japonica), J. Agric. Food Chem., 53, 1440-1444.

Yano, Y., Satomi, M. and Oikawa, H. (2006). Antimicrobial effect of spices and herbs on Vibrio parahaemolyticus, Int. J. Food Microbiol., 111, 6-11.

Zhang, Q.F., Jiang, Z.T. and Li, R. (2007). Complexation of allyl isothiocyanate with beta-cyclodextrin and its derivatives and molecular microcapsule of allyl isothiocyanate in beta-cyclodextrin, Eur. Food Res. Technol., 225, 407-413. 